\title{
BMJ Open Healthy Dads, Healthy Kids UK, a weight management programme for fathers: feasibility RCT
}

\author{
Tania Griffin, ${ }^{1}$ Yongzhong Sun, ${ }^{2}$ Manbinder Sidhu, ${ }^{3}$ Peymane Adab, ${ }^{4}$ \\ Adrienne Burgess, ${ }^{5}$ Clare Collins, ${ }^{6}$ Amanda Daley, ${ }^{7}$ Andrew Entwistle, ${ }^{8}$ \\ Emma Frew, ${ }^{9}$ Pollyanna Hardy, ${ }^{2}$ Kiya Hurley, ${ }^{10}$ Laura Jones (D),${ }^{4}$ Eleanor McGee, ${ }^{11}$ \\ Miranda Pallan, ${ }^{4}$ Myles Young, ${ }^{12}$ Philip Morgan, ${ }^{12}$ Kate Jolly ${ }^{4}$
}

To cite: Griffin T, Sun Y, Sidhu M, et al. Healthy Dads, Healthy Kids UK, a weight management programme for fathers: feasibility RCT. BMJ Open 2019;9:e033534. doi:10.1136/ bmjopen-2019-033534

- Prepublication history and additional material for this paper are available online. To view these files, please visit the journal online (http://dx.doi org/10.1136/bmjopen-2019033534).

Received 09 August 2019 Revised 03 October 2019 Accepted 01 November 2019

Check for updates

(c) Author(s) (or their employer(s)) 2019. Re-use permitted under CC BY. Published by BMJ.

For numbered affiliations see end of article.

Correspondence to

Professor Kate Jolly;

c.b.jolly@bham.ac.uk

\section{ABSTRACT}

Objective To assess (1) the feasibility of delivering a culturally adapted weight management programme, Healthy Dads, Healthy Kids United Kingdom (HDHK-UK), for fathers with overweight or obesity and their primary school-aged children, and (2) the feasibility of conducting a definitive randomised controlled trial (RCT).

Design A two-arm, randomised feasibility trial with a mixed-methods process evaluation.

Setting Socioeconomically disadvantaged, ethnically diverse localities in West Midlands, UK.

Participants Fathers with overweight or obesity and their children aged $4-11$ years.

Intervention Participants were randomised in a 1:2 ratio to control (family voucher for a leisure centre) or intervention comprising 9 weekly healthy lifestyle group sessions.

Outcomes Feasibility of the intervention and RCT was assessed according to prespecified progression criteria: study recruitment, consent and follow-up, ability to deliver intervention, intervention fidelity, adherence and acceptability, weight loss, using questionnaires and measurements at baseline, 3 and 6 months, and through qualitative interviews.

Results The study recruited 43 men, $48 \%$ of the target sample size; the mean body mass index was $30.2 \mathrm{~kg} /$ $\mathrm{m}^{2}$ (SD 5.1); $61 \%$ were from a minority ethnic group; and $54 \%$ were from communities in the most disadvantaged quintile for socioeconomic deprivation. Recruitment was challenging. Retention at follow-up of 3 and 6 months was $63 \%$. Identifying delivery sites and appropriately skilled and trained programme facilitators proved difficult. Four programmes were delivered in leisure centres and community venues. Of the 29 intervention participants, 20 $(69 \%)$ attended the intervention at least once, of whom $75 \%$ attended $\geq 5$ sessions. Sessions were delivered with high fidelity. Participants rated sessions as 'good/very good' and reported lifestyle behavioural change. Weight loss at 6 months in the intervention group $(n=17)$ was $2.9 \mathrm{~kg}(95 \% \mathrm{Cl}-5.1$ to -0.6$)$.

Conclusions The intervention was well received, but there were significant challenges in recruitment, programme delivery and follow-up. The HDHK-UK study was not considered feasible for progression to a full RCT based on prespecified stop-go criteria. Trial registration number ISRCTN16724454.
Strengths and limitations of this study

- This unique study provides new evidence into the feasibility of delivery of a weight management programme targeting fathers from socioeconomically deprived, ethnically diverse community settings, and its acceptability in this target population, a key strength given the relative paucity of research in this area.

- Recruitment methods were wide ranging, raising awareness of the study in a number of community settings; however, despite extensive efforts, recruitment to the study was a key challenge.

- The intention was to recruit male facilitators to deliver the intervention sessions. However, it was difficult to identify suitable facilitators with the necessary skill set; therefore, a range of male and female facilitators were recruited.

- Identifying a convenient time to run the intervention course to suit fathers, venue and facilitator availability proved challenging.

\section{INTRODUCTION}

Overweight and obesity remain a public health challenge and priority. ${ }^{1}$ At an individual level, excess weight results in fewer disease-free years and lowered life expectancy, $^{2}{ }^{3}$ and at a population level, obesity prevalence is associated with major economic burden. ${ }^{4}$

In England, overweight and obesity levels are higher in men compared with women with socioeconomic inequalities evident. ${ }^{5}$ Men from the lowest income quintile have a greater mean waist circumference than men from the highest income quintile. ${ }^{6}$ South Asian men have higher body fat percentage than white Europeans at the same body mass index (BMI). ${ }^{7}$

The transition to fatherhood has been identified as a critical period during which men are more likely to adopt obesogenic behaviours and are more susceptible to 
weight gain. ${ }^{8}$ While there have been several successful men-only weight management programmes, ${ }^{10-14}$ attendance to such activities remains higher among women. ${ }^{13}$ Fathers are also under-represented in interventions targeting family health behaviours. ${ }^{15}$ This emphasises the need to identify programmes which fathers are keen to engage with.

Healthy Dads, Healthy Kids (HDHK) is a weight management programme for fathers of primary schoolaged children that also addresses the healthy eating and physical activity behaviours of their children. The programme, developed and delivered in Australia, was shown to be successful at achieving weight loss in fathers. ${ }^{1617}$ In the current study, the HDHK programme was adapted with the intention of making it culturally acceptable to a multiethnic UK population. ${ }^{18}$ The aim of this study was to assess the feasibility of delivering the adapted HDHK programme and the feasibility to conduct a future randomised controlled trial (RCT) in a socioeconomically deprived ethnically diverse UK setting.

\section{METHODS}

\section{Study design and participants}

This study was a two-arm, randomised feasibility trial with a mixed-methods process evaluation. It was conducted in two urban local authority areas of the West Midlands, UK (site 1 and site 2) selected for their population profile and interest in supporting the programme. In 2017, both areas were ranked in the most deprived $20 \%$ of areas in the UK, with high ethnic diversity. ${ }^{19}$

The study aimed to recruit men who were father/stepfather/father figures (herein referred to as 'fathers') of primary school children (aged 4-11 years), aged 18-65 years with a BMI of $\geq 25 \mathrm{~kg} / \mathrm{m}^{2}\left(\geq 23 \mathrm{~kg} / \mathrm{m}^{2}\right.$ for minority ethnic groups $)^{7}$ and $/$ or a waist circumference of $\geq 94 \mathrm{~cm}$ (37 inches) who were willing to lose weight. Fathers did not have to be coresident with their children. Men were not eligible if they had a history of angina or other cardiovascular disease; had orthopaedic or joint problems that would be a barrier to vigorous physical activity; had weight loss of $3 \mathrm{~kg} / 7 \mathrm{lb}$ in the previous 3 months; had current diabetes and were not confident in managing their condition during exercise; were unable to speak and/ or understand English; and were involved in ongoing custody or access disputes and/or any contexts with a risk of domestic violence.

\section{Sample size}

As a feasibility study, no formal sample size calculation was performed. We aimed to recruit 90 men and their children to estimate the recruitment, follow-up and questionnaire completion rates to within $\pm 10 \%$ with $95 \%$ CIs, based on a worst case estimate of $50 \%$.

\section{Recruitment and randomisation}

Fathers were recruited (September 2017-January 2018) by the research team who had extensive experience of participant recruitment in a community setting. A range of methods were used over the recruitment period, including flyer distribution and promotion stands at leisure, community and shopping centres, places of worship and large workplace organisations. Recruitment via schools conducted through presentations at school assemblies and teacher meetings, stands at parent evenings, flyer distribution and talking to parents at school pick-up time. The study was promoted on social media (Twitter and Facebook).

Written informed consent was obtained from fathers and assent from children aged 8 years and over. Once baseline data were collected, participants were randomised (1:2 allocation ratio) to the control group (voucher for a single family visit to a leisure centre) or intervention group (adapted HDHK-UK programme). Randomisation was stratified by the father's ethnicity (white British or Irish/other ethnic group) and conducted using an automated online form developed by the University of Birmingham Clinical Trials Unit.

\section{Intervention}

The HDHK-UK intervention comprised weekly $90 \mathrm{~min}$ sessions over nine consecutive weeks; four courses were delivered. Fathers and children attended all sessions, which followed the same structure: 15 min discussion and review of the weekly activities followed by $30 \mathrm{~min}$, where children and fathers took part in an education session separately. The groups were facilitated by local, experienced and trained staff to ensure the sessions were interactive and discussion was encouraged. Facilitators were selected based on their experience of delivering group programmes and delivering health advice; they included health trainers (who provide community support for health-related behaviour changes) and sports coaches. They completed HDHK delivery training with either the Fatherhood Institute or the research team. The training included practising delivery of parts of the intervention. Fathers' sessions covered a range of lifestyle behaviours around the importance of physical activity, nutrition and parenting. Children were taught about healthy eating, physical activity and how to be a supportive family member by encouraging and modelling healthy lifestyle behaviours at home. The final $45 \mathrm{~min}$ of the session were spent doing physical activity within family groups. These practical sessions had three elements: 'rough and tumble' play; teaching children fundamental movement skills (catching, throwing and kicking); and aerobic fitness. The intervention is summarised in the template for intervention description and replication(TIDieR)checklist (online supplementary table 1). The HDHK intervention draws on concepts from family systems theory ${ }^{2021}$ and social cognitive theory ${ }^{22}$; the theoretical constructs are reported for the original programme ${ }^{17}$ Adaptations to the Australian resources were made by the research team (KJ and MSS) and one of the study partners (the Fatherhood Institute) in conjunction with the wider research team. The adaptations were informed by qualitative research 
with fathers and mothers from similar socioeconomic backgrounds and residing in the same geographical region. ${ }^{23}$ Adaptations focused on reducing the number of PowerPoint slides, simplifying and anglicising wording and updating the guidance and statistics to align to UK public health recommendations. References to foods, activities and images were updated to reflect a multicultural UK population. Fathers and their children attended every session and mothers were invited to one session when family food was discussed.

\section{Data collection and outcomes}

Data were collected from participants by trained researchers at baseline and at 3 and 6 months later in their home (or convenient location). Sociodemographic characteristics were collected by questionnaire. Based on postal code of residence, the Index of Multiple Deprivation (IMD) was used to determine socioeconomic status. ${ }^{24}$

\section{Primary outcomes: feasibility measures}

The outcomes relating to feasibility of delivery of HDHK-UK were the ability to recruit and retain facilitators, the ability to deliver sessions at a time and location convenient for participants, fidelity of delivery and acceptability to participants.

The outcomes relating to the feasibility of conducting a future definitive RCT were recruitment rate, willingness to be randomised, follow-up rates, level of completion of follow-up questionnaires, and father's weight change in the intervention group.

\section{Process evaluation}

Process measures were collected by the research team to determine the feasibility of intervention delivery and study processes, fidelity of intervention delivery and participant acceptability. Sixteen session observations (minimum of two per intervention course) were completed to assess content delivery and participant engagement. Participants and facilitators were also asked to complete feedback forms after each session and evaluating the session content and delivery. Qualitative interviews were conducted with 12 participants postintervention. One participant was interviewed at both 3 and 6 months. The average interview duration was $16 \mathrm{~min}$, and interviews were conducted either face-to-face $(n=4)$ or by telephone $(n=9)$. All facilitators $(n=7)$ were interviewed; five interviews were conducted individually, one with two facilitators together.

\section{Secondary outcome measures}

Secondary outcome measures for fathers were outcomes that would be collected in the main trial (if progressed to). These comprised percentage losing $\geq 5 \%$ body mass, change in waist circumference and percent body fat, self-reported physical activity (using the IPAQ-short, ${ }^{25}$ objectively measured physical activity (by a wrist-worn GeneActiv (Activinsights, Cambs, UK), triaxial accelerometer worn for 7 days on the father's non-dominant wrist), self-reported dietary intake (using food frequency items
(Food Frequency Questionnaire (FFQ)), father-child relationship outcomes (using the Parent-Child Relationship Questionnaire) ${ }^{26}$ parenting for physical activity (using the Parenting Strategies for Eating and Activity Scale $^{26}$ and the Physical Activity Modelling Subscale of the Activity Support Scale for Multiple Groups. ${ }^{27}$

Secondary outcome measures collected from the children comprised BMI z-score change (calculated using the LMS method ${ }^{28}$ and UK reference data ${ }^{29}$ ); percent body fat, overweight or obese, objectively measured physical activity (GeneActiv, worn by the eldest child only); parent-reported dietary intake for the eldest child (using the Family Nutrition and Physical Activity Questionnaire ${ }^{30}$ ); and behaviour and emotional well-being (using the Strength and Difficulties Questionnaire). ${ }^{31}$

To assess the feasibility of collecting outcome data for a future health economic analysis, we collected the EQ-5D-5L ${ }^{32}$ and ICEpop CAPability Measure for Adults ${ }^{33}$ from fathers and the Child Health Utility-9D ${ }^{33-35}$ from the children.

\section{Progression criteria}

Progression criteria, agreed on by the funding panel and the Study Steering Committee, were predefined to help evaluate whether the feasibility trial should be recommended to progress to a fully powered RCT. These are detailed in the Results section.

\section{Data analysis}

For the quantitative analyses, the aim was to assess the progression criteria and the feasibility of delivery of a main trial. All analyses were by intention-to-treat and were undertaken in STATA V.12. Feasibility outcomes are presented overall and by group with counts, percentages and $95 \%$ CIs. Weight changes in fathers at follow-up of 3 and 6 months are summarised using means, SD and withingroup $95 \%$ CIs. Baseline characteristics are summarised by group and overall, and the proposed secondary outcomes of a definitive trial are summarised by group only. Categorical data are presented using counts and percentages, and continuous data are presented using means and SDs or medians and IQRs, where appropriate. Since the study was not powered to detect treatment effects on clinical outcomes, p values and 95\% CIs were not reported.

Audio recordings of qualitative interviews were transcribed verbatim, anonymised and analysed using the framework approach. ${ }^{36}$

\section{Public involvement}

A public and patient participation group of eight fathers and two mothers from one of the research sites was involved throughout all stages of the study by contributing to decisions about outcome measures, commenting on intervention materials (in particular, the need for their simplification) and participant facing documents (downplaying the focus on weight management and focussing on the opportunity for father-child interaction 


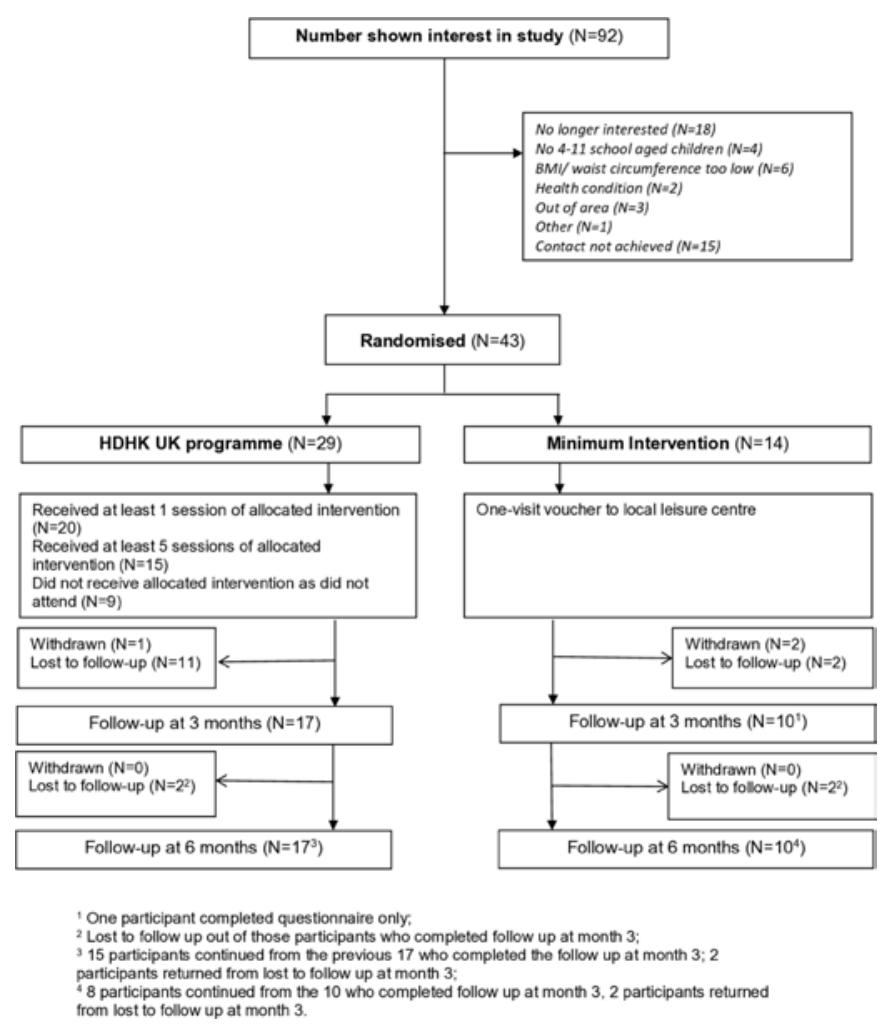

Figure 1 Participant flow through HDHK-UK for fathers. BMI, body mass index; HDHK-UK, Healthy Dads, Healthy Kids United Kingdom.

in recruitment materials), and advising on recruitment strategies and dissemination of the findings.

\section{RESULTS}

\section{Feasibility of conducting a definitive RCT}

Despite multiple recruitment methods, only 92 men expressed interest in the study, of whom 43 were recruited and randomised (intervention group, $\mathrm{n}=29$ ) (figure 1); 62 children were recruited. No participants declined randomisation after an assessment visit, nor was randomisation given as a reason for declining after expressing an interest in the study.

Four HDHK-UK courses were delivered, one at site 1 (in a youth centre) and three at site 2 (two in a leisure centre and one in a community centre).

\section{Baseline characteristics of participants}

The fathers' mean BMI at baseline was $30.2 \mathrm{~kg} / \mathrm{m}^{2}$ (SD 5.1 ), and their mean age was 40.0 years (range 23.8-56.0). The mean age of their participating children $(n=62)$ was 7.7 years (range $4.0-11.7$ ), of whom 20 (32.8\%) were overweight or obese. Overall, $60.5 \%$ of the participants were from minority ethnic groups, and $74.4 \%$ lived in the two most deprived quintiles of the IMD. Details of the baseline characteristics are presented in table 1 .

\section{Follow-up rates}

The follow-up rate at 3 and 6 months postintervention was $62.8 \%(n=27): 58.6 \%(n=17)$ in the intervention group and $71.4 \%(\mathrm{n}=10)$ in the control group (figure 1). Participants lost to follow-up at 6 months were more likely to be white British (online supplementary table 2).

Level of completion of follow-up questionnaires

Researchers experienced challenges in arranging appointments for data collection. Despite repeated reminders, researchers often arrived at a family's house to find they were no longer available. Work and after-school activities made finding times for recruitment visits difficult and impacted recruitment numbers. For fathers and their children who completed data collection appointments, the processes worked well. In the main, the completeness of the data was acceptable with the exception of high levels of missing data for the IPAQ questionnaire and fathers' waist circumference, which was frequently refused (23\% missing at baseline).

\section{Fathers' weight change in the intervention group}

Table 2 summarises fathers' weight change from baseline. The intervention group had a $2.9 \mathrm{~kg}(95 \%$ CI 0.6 to 5.1) reduction in weight at 6 months' follow-up.

\section{Secondary outcomes}

Secondary outcomes for fathers are summarised in table 2, and those for children are summarised in table 3. The intervention group of fathers had favourable reduction in waist circumference; $31 \%(\mathrm{n}=9)$ achieved a $5 \%$ reduction in body mass at 6 months. There were no serious adverse events requiring hospital admission or adverse events requiring medical attention during the intervention.

\section{Feasibility and acceptability of programme delivery}

Ability to recruit and retain facilitators

Site 1 provided health trainers employed by the local authority for the fathers' education component, and an independent fitness trainer delivered the physical activity elements. At site 2, two courses were delivered in a leisure centre by their staff, and one course was delivered in a community centre by sports coaches employed by a coaching organisation.

We experienced challenges in delivering training due to loss of potential facilitators as a result of rapid turnover of staff from various delivery organisations. Three forms of training were delivered: a 2-day HDHK training workshop delivered by the Fatherhood Institute, which had previously been conducted by the Australian team; a half day of top-up training by the Fatherhood Institute; and more flexible training for facilitators recruited after these sessions, delivered by a member of the research team (TLG) who had previous experience in coaching training.

We intended that two courses would be delivered at each site. However, due to organisational restructuring, the health trainers were only able to deliver one course.

\section{Challenges to recruiting participants}

There were significant challenges with recruitment. We obtained permission from 40 organisations to recruit 
Table 1 Baseline characteristics for fathers and children by treatment arm

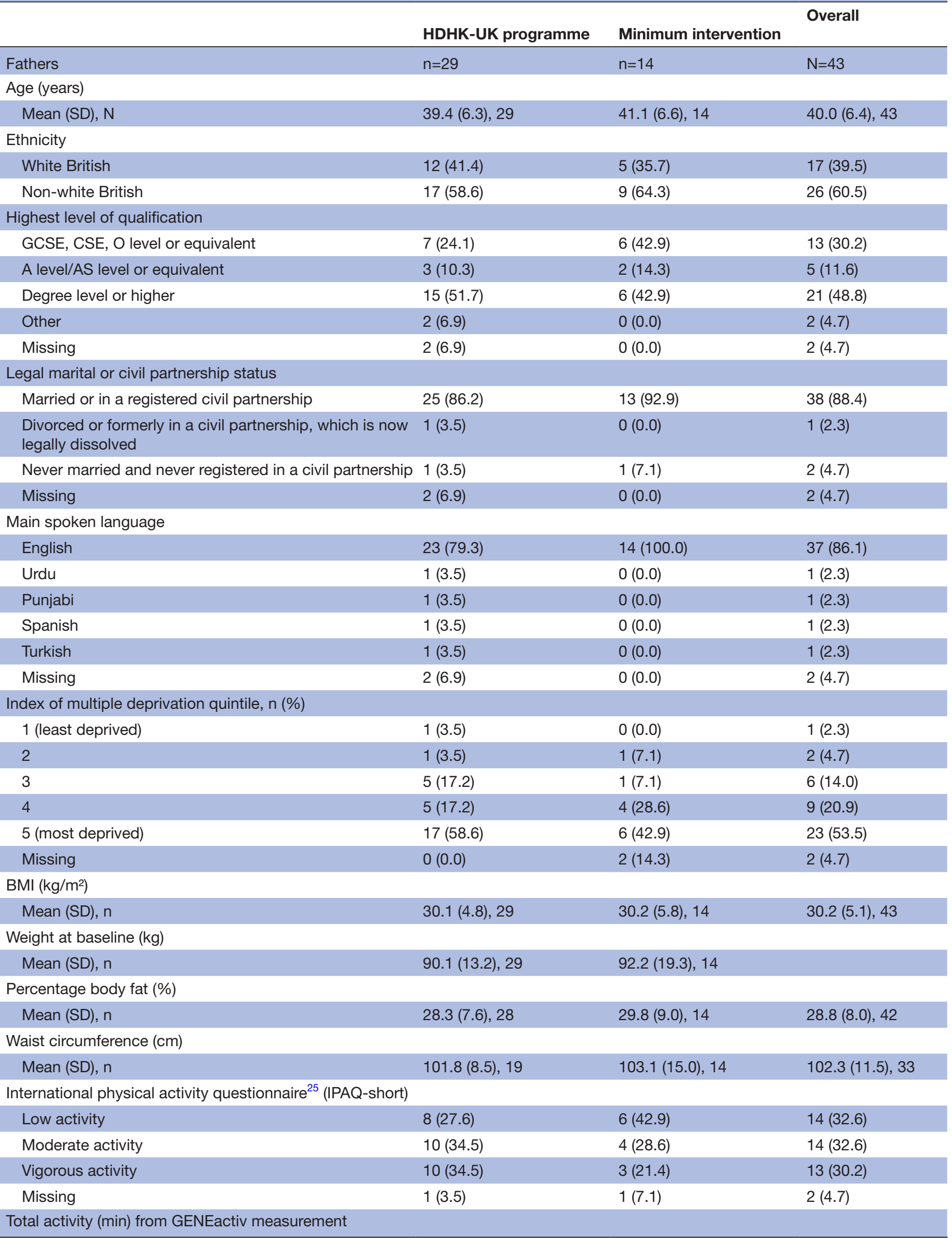


Table 1 Continued

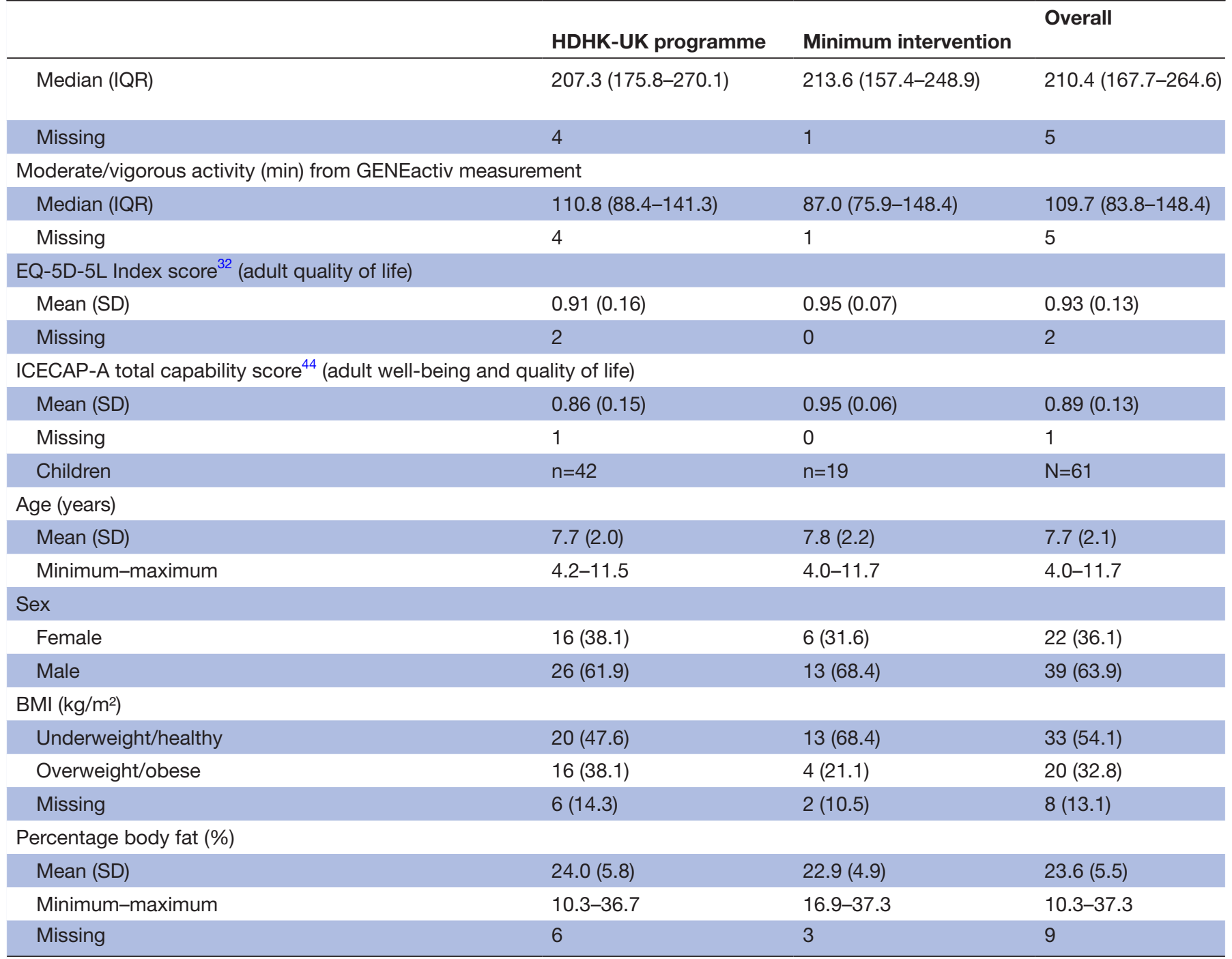

Note: All figures presented are $\mathrm{n}(\%)$ unless otherwise specified.

A level, Advanced level; AS level, Advanced Subsidiary level; BMI, body mass index; CSE, Certificate of Secondary Education; EQ-5D5L, EuroQol; GCSE, General Certificate of Secondary Education; HDHK-UK, Healthy Dads, Healthy Kids United Kingdom; ICECAP-A, ICEpop CAPability Measure for Adults; O level, Ordinary level.

on their premises (including 11 schools, 7 faith organisations and 6 sport/leisure centres), but it was a timeconsuming process. Communication with schools was slow and recruitment via children and 'mothers' was not a successful strategy. Fathers were hard to engage during recruitment activities, and session timings were often not convenient for their family. Additionally, as a result of a change in sites after funding was awarded (due to a local site withdrawing funding for adult weight management services), the sites involved considerable researcher travel time.

Deliver sessions at a time and location convenient for participants Aligning venue, facilitator and participant availability was a major challenge and resulted in considerable delays in commencing programme delivery. Evening sessions were difficult due to fathers' work commitments and children's meal and bed times. Although weekends were reported to be convenient by participants, the availability of facilitators and community venues was more limited.

I wasn't finishing work until 5 o'clock and then I was having to fight my way through the traffic to get there. If it had been any later, it's harder for the kids then because they've got to get up for school the next week. ... and weekends wouldn't be any good because the leisure centre would be packed. (ID B-008)

Session timings were also the main reason given by participants for non-attendance at intervention sessions.

It started at half past five which is obviously rush hour time. Dads are leaving work ... In the end, what happened was it went from a group of six dads and their children to the last session being just three dads .... 
Table 2 Weight change and secondary outcome measures for fathers by treatment arm

3 months

HDHK-UK programme Minimum intervention $(n=29)$

$(n=14)$
6 months

HDHK-UK programme Minimum intervention

$(n=29)$ $(n=14)$
Weight change from baseline
$(\mathrm{kg})$

Mean (SD), $n$

$(95 \% \mathrm{Cl})$

$-1.8(2.5), 17$

$(-3.1$ to -0.5$)$

(n=29)

Change from baseline in waist circumference $(\mathrm{cm})$

Mean (SD), $\mathrm{n} \quad-10.8(18.0), 9$

Change from baseline in $\%$ body fat

Mean (SD), $\mathrm{n}$

Mean (SD), $\mathrm{n} \quad-1.5(3.1), 16$
Physical activity measured by a GENEactive accelerometer

$-1.2(3.3), 9$

(-3.7 to 1.3$)$

$-2.9(4.1), 15$

$(-5.1$ to -0.6$)$

-2.0 (3.6), 10

$(-4.6$ to 0.6$)$

(IQR), $n$

$208.8 \quad 168.3$

3.4 (9.3), 6

$-5.2(5.0), 6$

$-2.8(6.4), 5$

Median for moderate/vigorous 113.3

activity (IQR), $\mathrm{n}$

(99.3-151.3), 17

(147.8-194.0), 9

(194.3-287.0), 11

(125.0-230.6), 9

$84.2 \quad 125.6$

68.2

EQ-5D-5L ${ }^{32}$ (adult health related quality of life)

Mean (SD), $n \quad 0.87$ (0.19), 17

(69.4-106.2), 9

(101.3-163.1), 11

(59.1-86.5), 9

ICECAP-A $^{34}$ (adult capability and well-being)

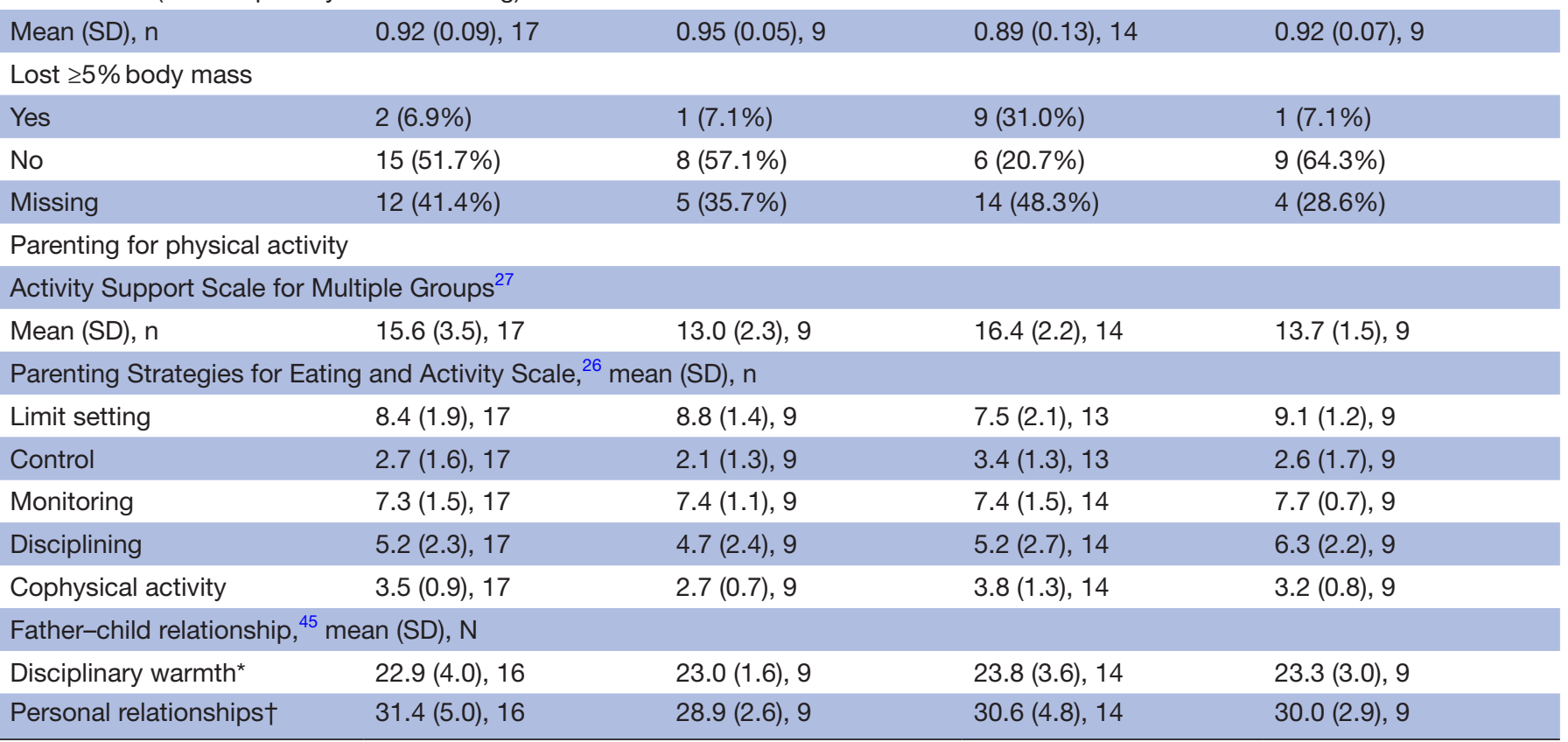

${ }^{*}$ Disciplinary warmth=praise + shared decision making +rationale

†Personal relationships (prosocial+intimacy+nurturance+companionship).

BMI, body mass index; EQ-5D-5L, EuroQol; ICECAP-A, ICEpop CAPability Measure for Adults.

I think that wasn't due to their motivation but their work time commitments. (ID B-068)

\section{Fidelity of delivery}

The intervention was delivered with high fidelity. The style of delivery and the small group sizes meant group discussions were encouraged, allowing content to be tailored to the group's needs (facilitators were trained to manage group discussions as part of the HDHK delivery training); however, facilitators reported finding it challenging to deliver all the session contents in the allocated time. This was especially difficult if participants arrived to the sessions late and facilitators had to balance content delivery while also allowing for group discussion and interaction. Researchers sometimes observed some session content being skipped or a delay in the start of the subsequent practical session. Despite the challenges, the facilitators ensured key content was delivered, which was verified in participant interviews, where they spoke of the key messages the course had focused on. 
Table 3 Secondary outcome measures for eldest child by treatment arm

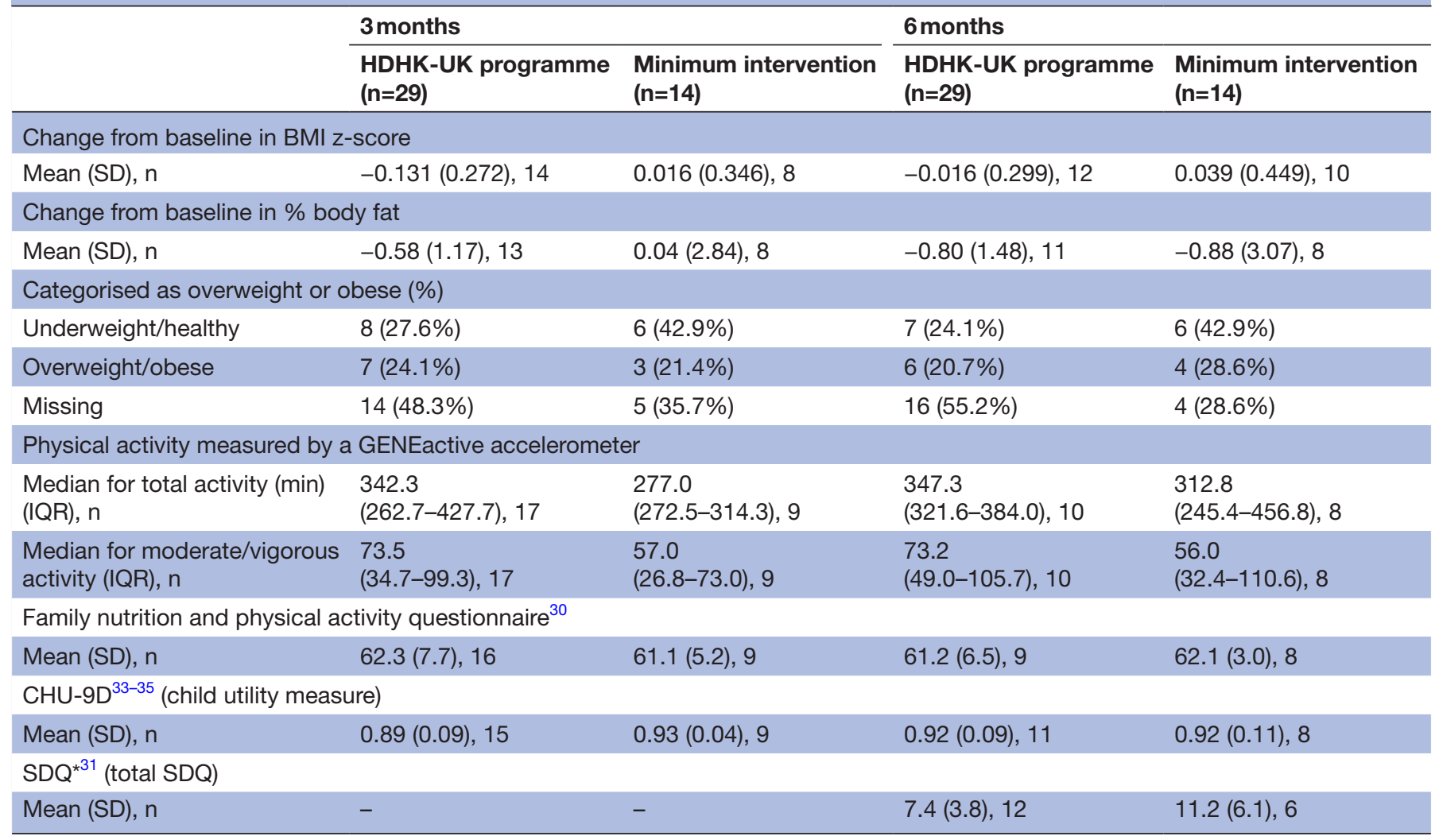

*SDQ: Each 1-point increase in the total difficulties score corresponds with an increase in the risk of developing a mental health disorder. BMI, body mass index; CHU-9D, Child Health Utility-9D; SDQ, Strengths and Difficulties Questionnaire.

Acceptability of adapted intervention to participants

Twenty participants $(69 \%)$ attended an intervention course at least once; 15 attended $(52 \%)$ at least five sessions. Fathers who attended the sessions and took part in a qualitative interview were positive about their experiences. Feedback sheets, observations and qualitative interviews with both participants and facilitators consistently showed high levels of acceptability towards the intervention programme.

It [HDHK-UK programme] was brilliant overall. I really enjoyed it. The kids enjoyed it. (ID B-068)

The group sessions were appreciated by participants:

I enjoyed the group-based elements ... it allowed to people to bounce off, talk about - and also the blokes, a little bit of competitive edge, especially when the weight round was coming. So I think it benefited me and I preferred the group format rather than just individually between me and my children. (ID A-077)

A strong theme was the appreciation of time spent with their children: 'I'm working during the week and it's just nice to have that dad-and-daughter time when just for a couple of hours it was just us and I think we've really benefited from that' (ID A-072).

Participants spoke highly of the facilitators for the education sessions: 'Fantastic, very well presented, well engaging, knowledgeable' (ID A-058) and the physical activity sessions: 'they were excellent, really good lads... we were always busy, always sweating, it was all good' (ID B-089). Similarly, the facilitators spoke positively of the programme.

A key challenge was delivery over the UK winter:

The weather had been against us a lot of the times, I think if we had done it now in the summer, I think it would have been a lot better for us (ID B-089)

....although we've been out Saturdays and Sundays it's trying to fit it into darker nights when it's been cold and it's been wet or it's been snowing or whatever else, that's been difficult. (ID A-072)

\section{Progression criteria}

Progression criteria for a full trial (table 4) were met for intervention acceptability to participants who attended and fidelity of delivery; recruitment to the trial was low and attrition was high $(37.2 \%, \mathrm{n}=16)$. Mean weight loss in the intervention arm was marginally below the prespecified $\geq 3 \mathrm{~kg}$ in the participants who were followed-up.

\section{DISCUSSION}

The aims of the current study were to assess the feasibility of delivering a culturally adapted HDHK programme in a 
Table 4 Progression criteria for a full trial

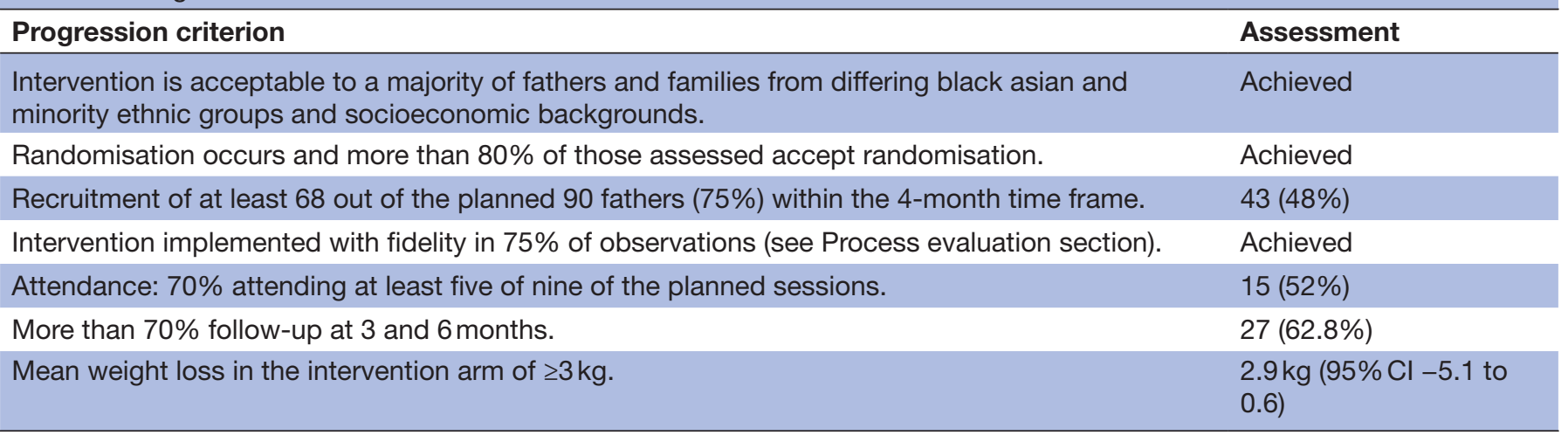

socioeconomically deprived UK setting and the feasibility of conducting a definitive RCT. Overall, it was possible to recruit and train facilitators to deliver the intervention programme with high fidelity. The programme was rated highly by the attending participants and delivery teams, and those who participated achieved weight loss comparable with other studies. ${ }^{13} 16$ However, recruitment of participants was difficult, and aligning participant, venue and facilitator availability to deliver the programme was a major challenge. As such, participant recruitment and attendance rates were low and the progression criteria for the study were not met.

Despite multiple strategies, encouraging fathers to engage with the study was challenging. Attempts to enhance recruitment through schools, an approach used in the Australian setting, proved more difficult than anticipated. Schools are often involved in multiple projects and programmes, which may have meant they felt they did not have added capacity for HDHK-UK. It was recognised during recruitment that there can be sensitivities around discussing weight. To try to offset this, the opportunity for fathers to spend time with their children was emphasised. Challenges in recruitment have also been recognised in a similar study with fathers ${ }^{37}$ and as a common theme in a systematic review of weight loss trials in men. ${ }^{13}{ }^{14}$ However, the findings and experiences from this current study differ from those of the Australian HDHK RCT. ${ }^{16}{ }^{17}$ Notably, the Australian study used a wait-list study design where all families were guaranteed to receive the programme at some point, which may have been more appealing for participants. An additional difference was a greater availability of sessions.

Nearly a third of participants allocated to the intervention group did not engage with the programme. This has been seen in other group-based health-related programmes ${ }^{38-40}$ but differs from the HDHK trial in Australia. ${ }^{1617}$ The primary reason given for non-attendance or non-completion of the programme was that the session timings were not suitable, which was corroborated by the research team, which found identifying a convenient time for programme delivery to be one of the most significant challenges of the study. Evening sessions had to align to fathers' working patterns and children's school and bedtime routines, resulting in sessions often being set when traffic was at its heaviest. This was compounded by running the sessions in the winter with dark nights and poor weather. Weekend sessions often clashed with family activities, and venue and facilitator availability were more limited. Poor weather is likely to have also impacted recruitment, especially at schools where parents were not keen on stopping to talk to the study team in rainy or cold conditions. Delivery in summer and offering more choice of sessions might mitigate some of these challenges.

The facilitators reported it to be challenging to deliver the full content of the father's education session within the allocated $30 \mathrm{~min}$, exacerbated by participants arriving late. The approach to sessions was to encourage interaction and discussion, which was appreciated by participants and necessary if the group size was small. However, this at times challenged the fidelity of delivery, and if the programme were to be rolled out, we would recommend reducing session content further.

The youngest children participating in the current study had difficulty in engaging with written materials; 4-year-olds were not eligible in the Australian studies. ${ }^{161740}$

\section{Strengths and limitations}

The study has a number of strengths. While successful weight management trials in men have been reported, ${ }^{1012131617}$ most participants in weight loss trials are women. ${ }^{41}{ }^{42}$ In addition, very few specifically target ethnic minority population groups ${ }^{41}$ or recruit from socioeconomically disadvantaged populations, which is a unique contribution of this study.

This study also had limitations. First, the small number of participants recruited and attending the programme meant some adaptation for a smaller group size was required, but meant some of the group atmosphere was reduced. Those who attended enjoyed the programme, and while the remainder advised that session timing was their main reason for non-attendance, the small sample size created bias, especially for the qualitative interviews. While every attempt was made to interview non-attendees and non-completers, engagement was low. We had also planned to interview participants with their partners and children present, but logistically, this proved difficult to 
arrange; many of the children were too young to participate in an interview, and most fathers opted for a phone interview as they found this more convenient.

Another limitation was the change in facilitation staff throughout the study, resulting in the need to run individualised training sessions after the original training delivered by the Fatherhood Institute. While the additional training was delivered by an experienced researcher (TLG), the process presented a significant time commitment and may have led to differences in delivery style.

Due to the study timeline and working with school terms and avoiding Ramadan, the intervention was delivered at the height of winter, a contrast to the Australian programme, which is delivered only in summer months. This is likely to have impacted both recruitment and intervention attendance.

The session observations and feedback from participants and facilitators were overwhelmingly positive. Despite the acknowledged limitations, the adapted HDHK programme was delivered and received well. The observed outcome measure of weight change across the programme was promising, and the data collected showed good levels of completion.

\section{Future research}

At this time, the trial steering committee did not recommend progressing to a full RCT in the context in which this study was set. However, there may be other settings within the UK in which some of the barriers that were faced either would not occur or could not be addressed or ameliorated. Another avenue which could be explored would be for some of the education sessions to be delivered online, which has been shown to be successful in some groups ${ }^{43}$ and would reduce the time difficulties experienced in delivering content in the face-to-face sessions. In line with the recommendations from the study patient and public involvement (PPI) group, delivery of the programme outside the context of weight management may result in higher recruitment.

\section{CONCLUSIONS}

The majority of difficulties experienced in this study stem from the setting and context under which it was to be delivered, and while we do not recommend progressing this feasibility study to a full RCT, we recognise that the outcomes and recommendations made may have been different had the study been trialled in a different setting. A number of challenging circumstances converged within the study, which likely compounded further the difficulties encountered in delivery: recruitment difficulties, the untimely change in facilitation staff, and the poor weather throughout recruitment and delivery. The intervention was rated highly by those who attended, and the weight loss achieved by the intervention participants was promising, although no definitive conclusions can be drawn owing to the small sample size.

\section{Author affiliations}

${ }^{1}$ Department of Health, University of Bath, Bath, UK

${ }^{2}$ Birmingham Clinical Trials Unit (BCTU), University of Birmingham, Birmingham, UK ${ }^{3}$ Health Services Management Centre, University of Birmingham, Birmingham, UK

${ }^{4}$ Institute of Applied Health Research, University of Birmingham, Birmingham, UK

${ }^{5}$ Fatherhood Institute, Marlborough, UK

${ }^{6}$ School of Health Sciences, University of Newcastle, Callaghan, New South Wales, Australia

${ }^{7}$ School of Sport, Exercise and Health Sciences, Loughborough University,

Loughborough, UK

${ }^{8}$ Public member, Leamington Spa, UK

${ }^{9} \mathrm{Health}$ Economics Unit, Institute of Applied Health Research, University of Birmingham, Birmingham, UK

${ }^{10}$ Cancer Research UK Clinical Trials Unit, University of Birmingham, Birmingham, UK

${ }^{11}$ Birmingham Community Healthcare NHS Trust, Aston, UK

${ }^{12}$ School of Education, University of Newcastle, Callaghan, New South Wales, Australia

\section{Twitter Laura Jones @drlauraljones and Philip Morgan @philmorgo}

Acknowledgements We would like to thank all participating families and the local authorities for their support and direction and the facilitators who delivered the programme. We acknowledge members of the study steering committee: Professor John Wright, Bradford Institute for Health Research (Chair); Professor Pat Hoddinott, University of Stirling; Elaine Nicholls, Keele University; Mr Ray Fiveash, PPI representative. We acknowledge Kathy Jones and Cassius Campbell, who delivered the training programme, and Jeremy Davies from the Fatherhood Institute. We thank Jeszemma Garratt from the Fatherhood Institute and the Parent Advisory Panel and staff who contributed to the research delivery: Dr Khaled Ahmed, Felicity Brant, David Sardar and Meanaz Akhtar. We acknowledge academics from the University Newcastle, Australia, who developed the original Healthy Dads, Healthy Kids programme and who supported the study through the development of training materials and shared their experience of delivery in the Australian setting: Professor David Lubans and Kristen Saunders.

Contributors KJ, PA, AB, CC, AD, EF, LJ, MP, MS, MY and PM conceived the study; $\mathrm{KJ}$ was the principal investigator; $\mathrm{YS}$ undertook the statistical analysis; $\mathrm{PH}$ was the senior statistician; TG was the study coordinator and led the process evaluation; $\mathrm{LJ}$ was the qualitative lead; $\mathrm{TG}$ and $\mathrm{KH}$ undertook the qualitative interviews and analysis; PM and CC conceived and designed the original Healthy Dads, Healthy Kids intervention; PM and MY advised on training and delivery; CC and EM advised on dietary assessment; AE was the PPI lead; TG and KJ drafted the manuscript; all authors interpreted the findings, commented on paper drafts and agreed on the final version.

Funding Study funding was granted in October 2015 by the National Institute of Health Research (NIHR) Public Health Research programme (Ref 14/185/13); $\mathrm{KJ}$ is partly funded by NIHR Collaborations for Leadership and Health Research and Care West Midlands. The views expressed are those of the authors and not necessarily those of the NHS, the NIHR or the Department of Health and Social Care.

Competing interests PM and CC designed the original Healthy Dads, Healthy Kids Programme in Australia.

Patient consent for publication Not required.

Ethics approval Ethical approval for the two phases of the study was obtained from the University of Birmingham Science, Technology, Engineering and Mathematics Ethical Review Committee (16 January 2017, ethics reference; ERN_16-1323).

Provenance and peer review Not commissioned; externally peer reviewed. Data availability statement Data are available upon reasonable request.

Open access This is an open access article distributed in accordance with the Creative Commons Attribution 4.0 Unported (CC BY 4.0) license, which permits others to copy, redistribute, remix, transform and build upon this work for any purpose, provided the original work is properly cited, a link to the licence is given, and indication of whether changes were made. See: https://creativecommons.org/ licenses/by/4.0/.

\section{ORCID iD}

Laura Jones http://orcid.org/0000-0002-4018-3855 


\section{REFERENCES}

1 NHS. The NHS long term plan London, 2019.

2 Kopelman P. Health risks associated with overweight and obesity. Obes Rev 2007;8:13-17.

3 Prospective Studies Collaboration. Body-mass index and causespecific mortality in 900000 adults: collaborative analyses of 57 prospective studies. The Lancet 2009;373:1083-96.

4 Public Health England. Health matters: obesity and the food environment, 2017. Available: https://www.gov.uk/government/ publications/health-matters-obesity-and-the-food-environment/ health-matters-obesity-and-the-food-environment-2 [Accessed 28 May 2018].

5 National Statistics. Statistics on obesity, physical activity and diet. England 2017. National statistics, 2017. Available: https://files.digital. nhs.uk/publicationimport/pub23xxx/pub23742/obes-phys-acti-dieteng-2017-rep.pdf [Accessed 28 May 2018].

6 NHS Digital. Health survey for England 2016. adult overweight and obesity, 2016. Available: https://digital.nhs.uk/data-and-information/ publications/statistical/health-survey-for-england/health-survey-forengland-2016 [Accessed 28 May 2018].

7 National Institute for Health and Clinical Excellence. BMI: preventing ill health and premature death in black, Asian and other minority ethnic groups. Public health guideline [PH46], 2013.

8 Gettler LT, Sarma MS, Gengo RG, et al. Adiposity, CVD risk factors and testosterone: variation by partnering status and residence with children in US men. Evol Med Public Health 2017;2017:67-80.

9 Garfield CF, Duncan G, Gutina A, et al. Longitudinal study of body mass index in young males and the transition to fatherhood. $A m \mathrm{~J}$ Mens Health 2016;10:NP158-67.

10 Morgan PJ, Callister R, Collins CE, et al. The SHED-IT community trial: a randomized controlled trial of internet- and paper-based weight loss programs tailored for overweight and obese men. Ann Behav Med 2013;45:139-52.

11 Morgan PJ, Collins CE, Plotnikoff RC, et al. The impact of a workplace-based weight loss program on work-related outcomes in overweight male shift workers. J Occup Environ Med 2012;54:122-7.

12 Hunt K, Wyke S, Gray CM, et al. A gender-sensitised weight loss and healthy living programme for overweight and obese men delivered by Scottish premier League football clubs (FFIT): a pragmatic randomised controlled trial. Lancet 2014;383:1211-21.

13 Robertson C, Archibald D, Avenell A, et al. Systematic reviews of and integrated report on the quantitative, qualitative and economic evidence base for the management of obesity in men. Health Technol Assess 2014:18:1-424.

14 Young MD, Morgan PJ, Plotnikoff RC, et al. Effectiveness of maleonly weight loss and weight loss maintenance interventions: a systematic review with meta-analysis. Obes Rev 2012;13:393-408.

15 Morgan PJ, Young MD, Lloyd AB, et al. Involvement of fathers in pediatric obesity treatment and prevention trials: a systematic review. Pediatrics 2017;139:e20162635.

16 Morgan PJ, Collins CE, Plotnikoff RC, et al. The 'Healthy Dads, Healthy Kids' community randomized controlled trial: A communitybased healthy lifestyle program for fathers and their children. Prev Med 2014;61:90-9.

17 Morgan PJ, Lubans DR, Callister R, et al. The 'Healthy Dads, Healthy Kids' randomized controlled trial: efficacy of a healthy lifestyle program for overweight fathers and their children. Int $\mathrm{J}$ Obes 2011;35:436-47.

18 Jolly K, Griffin T, Sidhu M, et al. A weight management programme for fathers of children aged 4-11: cultural adaptation and the healthy Dads, healthy kids UK feasibility RCT. Public Health Research. in press.

19 Office for National Statistics. Local area report for areas in England and Wales, 2017. Available: https://www.nomisweb.co.uk/reports/ localarea [Accessed 09 Oct 2018].

20 Golan M, Weizman A. Familial approach to the treatment of childhood obesity: conceptual model. J Nutr Educ 2001;33:102-7.

21 Golan M, Weizman A, Apter A, et al. Parents as the exclusive agents of change in the treatment of childhood obesity. Am J Clin Nutr 1998:67:1130-5.

22 Bandura A, National Inst of Mental Health. Prentice-Hall series in social learning theory. social foundations of thought and action: a social cognitive theory. Englewood Cliffs, NJ, US: Prentice-Hall, Inc, 1986.
23 Jolly KGT, Sidhu M, Adab P, et al. A weight management programme for fathers: cultural adaptation of healthy Dads, healthy kids UK and feasibility RCT. Public Health Research in press.

24 Department for Communities and Local Government. The English indices of deprivation 2015. National statistics, 2015. Available: https://assets.publishing.service.gov.uk/government/uploads/ system/uploads/attachment_data/file/465791/English_Indices_of Deprivation_2015_-_Statistical_Release.pdf [Accessed 09 Oct 2018].

25 Craig CL, Marshall AL, Sjostrom M, et al. International physical activity questionnaire: 12 -country reliability and validity. Med Sci Sports Exerc 2003;35:1381-95.

26 Larios SE, Ayala GX, Arredondo EM, et al. Development and validation of a scale to measure Latino parenting strategies related to children's obesigenic behaviors. The parenting strategies for eating and activity scale (PEAS). Appetite 2009:52:166-72.

27 Lampard AM, Nishi A, Baskin ML, et al. The activity support scale for multiple groups (ACTS-MG): Child-reported physical activity parenting in African American and non-Hispanic white families. Behavioral Medicine 2016;42:112-9.

$28 \mathrm{Pan} \mathrm{H}$, Cole T. LMS growth, a Microsoft Excel add-in to access growth references based on the LMS methods. in. 2.77 ED, 2012.

29 McCarthy HD, Jarrett KV, Crawley HF. The development of waist circumference percentiles in British children aged 5.0-16.9 y. Eur $J$ Clin Nutr 2001;55:902-7.

30 Ihmels MA, Welk GJ, Eisenmann JC, et al. Development and preliminary validation of a family nutrition and physical activity (FNPA) screening tool. Int J Behav Nutr Phys Act 2009;6.

31 Goodman R. Psychometric properties of the strengths and difficulties questionnaire. J Am Acad Child Adolesc Psychiatry 2001;40:1337-45.

32 Herdman M, Gudex C, Lloyd A, et al. Development and preliminary testing of the new five-level version of EQ-5D (EQ-5D-5L). Qual Life Res 2011;20:1727-36.

33 Stevens KJ. Working with children to develop dimensions for a preference-based, generic, pediatric, health-related quality-of-life measure. Qual Health Res 2010;20:340-51.

34 Stevens K. Developing a descriptive system for a new preferencebased measure of health-related quality of life for children. Qual Life Res 2009;18:1105-13.

35 Stevens K. Assessing the performance of a new generic measure of health-related quality of life for children and refining it for use in health state valuation. Appl Health Econ Health Policy 2011;9:157-69.

36 Gale NK, Heath G, Cameron E, et al. Using the framework method for the analysis of qualitative data in multi-disciplinary health research. BMC Med Res Methodol 2013;13:117.

37 Mobley A, Gans K, Adamsons K, et al. Healthy fathers, healthy kids: feasibility of a Father-focused childhood obesity prevention program for low-income families with preschool aged children (OR13-03-19). Curr Dev Nutr 2019;3.

38 Jolly K, Lewis A, Beach J, et al. Comparison of range of commercial or primary care led weight reduction programmes with minimal intervention control for weight loss in obesity: Lighten up randomised controlled trial. BMJ 2011;343.

39 British Heart Foundation. The National audit of cardiac rehabilitation (NACR) annual statistical report 2017, 2018.

40 Pallan M, Griffin T, Hurley KL. Cultural adaptation of an existing children's weight management programme: the CHANGE intervention development and feasibility RCT. Health Technology Assessment, 2018

41 Pagoto SL, Schneider KL, Oleski JL, et al. Male inclusion in randomized controlled trials of lifestyle weight loss interventions. Obesity 2012;20:1234-9

42 NICE. Weight management: lifestyle services for overweight or obese adults: Public Health Guidleine [PH53], 2014.

43 Mensorio MS, Cebolla-Martí A, Rodilla E, et al. Analysis of the efficacy of an internet-based self-administered intervention ("Living Better") to promote healthy habits in a population with obesity and hypertension: An exploratory randomized controlled trial. Int $J$ Med Inform 2019;124:13-23.

44 Al-Janabi H, Flynn TN, Coast J. Development of a self-report measure of capability wellbeing for adults: the ICECAP-A. Qual Life Res 2012;21:167-76.

45 Furman W, Giberson R. Identifying the links between parents and their children's sibling relationships. In: Shulman S, ed. Human development, vol. 7. close relationships and socioemotional development. Westport, CT, US: Ablex Publishing, 1995: 95-108. 